Research Paper

\title{
HOXA5 and p53 cooperate to suppress lung cancer cell invasion and serve as good prognostic factors in non-small cell lung cancer
}

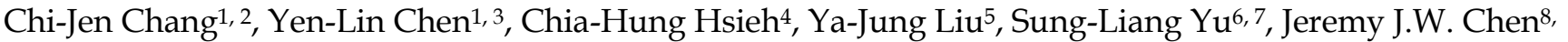 \\ ${ }^{9}$, Chi-Chung Wang ${ }^{\square}$ \\ 1. School of Medicine, Fu Jen Catholic University, New Taipei, Taiwan; \\ 2. Division of Pediatric Surgery, Department of Surgery, Shin Kong Wu Ho-Su Memorial Hospital, Taipei, Taiwan; \\ 3. Department of Pathology, Cardinal Tien Hospital, New Taipei, Taiwan; \\ 4. Graduate Institute of Biomedical Sciences, China Medical University, Taichung, Taiwan; \\ 5. Graduate Institute of Biomedical and Pharmaceutical Science, Fu Jen Catholic University, New Taipei, Taiwan; \\ 6. Department of Clinical and Laboratory Sciences and Medical Biotechnology, National Taiwan University College of Medicine, Taipei, Taiwan; \\ NTU Center of Genomic Medicine, National Taiwan University College of Medicine, Taipei, Taiwan; \\ 8. Institute of Biomedical Sciences, National Chung Hsing University, Taichung, Taiwan; \\ 9. Agricultural Biotechnology Center, National Chung Hsing University, Taichung, Taiwan.
}

$\square$ Corresponding author: Chi-Chung Wang, Ph.D., Graduate Institute of Biomedical and Pharmaceutical Science, Fu Jen Catholic University, New Taipei, 24205, Taiwan ROC. Phone: 886-2-29052039, Fax: 886-2-29053415, Email: 075006@mail.fju.edu.tw.

(C) Ivyspring International Publisher. This is an open access article distributed under the terms of the Creative Commons Attribution (CC BY-NC) license (https://creativecommons.org/licenses/by-nc/4.0/). See http://ivyspring.com/terms for full terms and conditions.

Received: 2016.08.22; Accepted: 2017.02.14; Published: 2017.04.09

\begin{abstract}
Lung cancer is the leading cause of cancer mortality worldwide and tumor metastasis is the major cause of cancer-related death. Our previous study suggested that Homeobox A5 (HOXA5) could inhibit lung cancer cell invasion via regulating cytoskeletal remodeling and involved in tumor metastasis. Recently, consensus HOX binding sites was found in the $\mathrm{p} 53$ gene promoter region. However, whether the HOXA5 could cooperate with $\mathrm{p} 53$ and contribute the inhibition of lung cancer cell invasion is still unclear. The aim of the current study is to elucidate the correlation of HOXA5 and p53 in tumor invasion and its prognostic influence in lung cancer patient specimens. Totally 71 cases of primary non-small cell lung cancer (NSCLC) were collected. The median follow-up period is 6.8 years. Immunohistochemical stain for p53 and HOXA5 were performed. Kaplan-Meier plot was done for overall survival analysis. In addition, lung cancer cell lines transfected with wild-type or mutated $\mathrm{p} 53$ constructs were overexpressed with HOXA5 for invasion assay. In human specimens, HOXA5 expressed mainly in the cytoplasm (54.1\%) rather than nuclei $(14.6 \%)$ of the NSCLC tumor part. The HOXA5 expression is higher in adenocarcinoma than in squamous cell carcinoma $(P<0.001)$. In addition, poor prognosis is seen in group with both non-immunoreactive for $\mathrm{p} 53$ and HOXA5. HOXA5 and $\mathrm{p} 53$ could cooperate to inhibit tumor cell invasion significantly partly by decreasing MMP2 activity in a concentration-dependent manner. Our studies provide new insights into how HOXA5 and p53 cooperate to contribute to the suppression of lung cancer cell invasion and play good prognostic roles in NSCLC.
\end{abstract}

Key words: HOXA5, p53, invasion, prognosis, lung cancer.

\section{Introduction}

Although lung cancer is not the most common cancer, it is the leading cause of cancer mortality worldwide. Lung cancer accounting for more than 1.6 million people received a new diagnosis, comprising $13 \%$ of all new cancer diagnoses, and 1.4 million died of lung cancer, which was $18 \%$ of all cancer deaths 
[1-4]. Pathologic assessment of lung cancers is a crucial component for the diagnosis, management, and prognosis of lung cancer [5,6]. In addition, prognostic factors are usually important for physicians and patients to realize the outcome of the treatment plan. Selective diagnostic techniques, including immunohistochemical panels, and decision analysis will not only increase diagnostic accuracy but also provide more information of prognosis $[1,7]$.

Homeobox transcription factors modulate signaling pathways controlling organ morphogenesis and maintain cell fate and differentiation in adults [8,9]. Recently, the involvement of Hox genes in organogenesis, especially in lung, has been discussed extensively $[10,11]$. The underlying mechanisms of HOXA5 in lung development may modulate by Notch signaling [12]. HOXA5 involves not only in normal lung development, but also in tumorigenesis. Several studies reported that the methylation status of HOXA5 gene may contribute to the tumor prognosis [13-15]. HOXA5 methylation is also presence in the lung cancer $[16,17]$. There is about $81.3 \%$ of non-small cell lung cancer (NSCLC) presence of HOXA5 promoter methylation. In addition, RT-PCR and immunohistochemical analysis showed that HOXA5 methylation correlates with gene expression [17]. Our previous study showed that the expression of HOXA5 is higher in less invasive cells than in highly invasive cells. In addition, overexpression of HOXA5 gene could inhibit the invasive ability of lung cancer cells [18]. Therefore, we suggested HOXA5 would be an invasive suppressor in NSCLC.

Expression of the p53 gene protects cells against malignant transformation [19-21]. Recently, consensus HOX binding sites in the p53 promoter was found and transient transfection of HOXA5 could activate the p53 promoter [22]. Loss of p53 expression in human breast cancer may be primarily due to the lack of HOXA5 expression [22]. Although our recent study suggested that HOXA5 might suppress lung cancer metastasis via cytoskeleton remodeling regulation [18], no further investigation was done to see the correlation between p53 and HOXA5 in NSCLC. The prognostic influence of p53 in NSCLC was reported and it is logical to hypothesize HOXA5, a tumor suppressor gene, may also associate with prognosis in NSCLC $[18,21,23]$. Thus, the aim of the current study is to investigate the expression of HOXA5 and p53 proteins in resected NSCLC samples by using immunohistochemical analyses and correlated these expression patterns with the lung cancer patients' clinicopathological characteristics. Furthermore, the combined effects of p53 and HOXA5 gene expression on cancer cell invasion will also be determined in NSCLC cell lines.

\section{Materials and Methods}

\section{Cell culture}

All cell lines, including H1299 (ATCC CRL-5803), NCI-H322M, and NCI-H522 (ATCC CRL-5810), were maintained at $37^{\circ} \mathrm{C}$ in a humidified atmosphere containing $5 \% \mathrm{CO}_{2}$. Cells were cultured in DMEM or RPMI 1640 medium (Life Technologies, Rockville, MD, USA) supplemented with $10 \%$ heat-inactivated fetal bovine serum (FBS; Life Technologies) and $1 \%$ penicillin-streptomycin (Life Technologies).

\section{Plasmids and transfection}

The HOXA5 expression plasmid pcDNA3.1-HOXA5 was constructed by cloning the full-length human HOXA5 cDNA from the previous study [18]. The expression vectors for human p53 wild-type and mutants were gifts from Dr. T.-M. Hong. All transfection experiments were carried out with Lipofectamine 2000 reagents (Invitrogen) according to the manufacturer's instructions.

\section{In vitro invasion assay}

The invasiveness of tested cells transfected with HOXA5, various p53 mutant constructs or vector alone was examined in a transwell assay using chambers $(8-\mu \mathrm{m}$ pore size; Corning Costar, Cambridge, MA, USA) and transwell filters coated with Matrigel (BD Biosciences, Franklin Lakes, NJ, USA), as described previously [18]. The number of cells attached to the lower surface of the polycarbonate filter was determined under a light microscope at $400 \times$ magnification.

\section{Gelatin zymography assay}

Cells were plated at a density of $5 \times 10^{5}$ cells/well in 6-well plates for 6 hours and then cultured in serum-free medium for another 16 hours. For MMP-2 activity detection, conditioned medium were prepared without boiling or reduction and subjected to SDS polyacrylamide gels containing $0.1 \%$ gelatin. After electrophoresis, the gels were washed with 2.5\% Triton X-100 for $30 \mathrm{~min}$ and incubated in a developing buffer (50 mM Tris-HCl, pH 8.0, 0.2 M NaCl, $5 \mathrm{mM}$ $\mathrm{CaCl}_{2}, 0.02 \%$ Brij35) at $37^{\circ} \mathrm{C}$ for 24 hours. Finally, the gel was stained with Commassie brilliant blue R-250.

\section{Patients}

We retrospectively randomly selected cases received thoracic surgery in two hospitals (SKWHSMH and CTH) between 2000 and 2003. All of these cases had no family history of lung cancer or malignancy in first-degree relatives as judged by interviews at the time of admission for surgery. All the patients with malignant disease had received 
routine pulmonary pneumonectomy, lobectomy or wedge resection. We further exclude subjects have been received chemotherapy or radiation therapy before resection. Finally, 71 cases of primary NSCLC were collected. All clinical charts and histopathology reports of patients were reviewed regarding age, gender, tumor size, location, histological subtype, differentiation grade, lymph nodes metastasis or distant metastasis and TMN staging. All cases were followed from 3 to 10 years. The median follow up period of study subjects is 6.8 years. The overall survival was also recorded. All enrolled cases were de-linked anonymously for protection and this study protocol was approved by the institutional review board of Shin Kong Wu Ho-Su Memorial Hospital and Cardinal Tien Hospital. Finally, by using the Kaplan Meier-plotter online database (GSE50081 cohort), another 181 patients with NSCLC was also applied in the prognosis analysis.

\section{Histology, immunohistochemistry and scoring}

The constructed tissue array paraffin embedded blocks were in $5 \mu \mathrm{m}$-thick sections to perform H\&E stain. In every case, carcinoma type, cell differentiation grade, growth pattern, tumor cell nuclear morphology, metaplasia, calcification, necrosis, mitosis count and other specific differentiation were re-checked by pathologist (YLC). IHC stains were performed, using the Ventana BenchMark XT automated stainer (Ventana, Tucson, AZ). Briefly, $4 \mu \mathrm{m}$-thick sections were consecutively from formalin-fixed, paraffin-embedded tissue. Sections were mounted on silanized slides and allowed to dry overnight at $37^{\circ} \mathrm{C}$. After deparaffinization and rehydratation, slides were incubated with 3\% hydrogen peroxide solution for 5 min. After a washing procedure with the supplied buffer, tissue sections were repaired for $40 \mathrm{~min}$ with ethylenediamine tetraacetic acid. The slides were again incubated with the primary antibody for 60 minutes at $37^{\circ} \mathrm{C}$ and then overnight at $4^{\circ} \mathrm{C}$. The primary antibodies, p53 (1:50, Novus) and HOXA5 (1:50, Abcam) were performed. After three rinses in buffer, the slides were incubated with the secondary antibodies (unbiotinylated antibody, EnVisionTM System, HRP, anti-mouse/rabbit, DakoCytomation). Tissue staining were visualized with a DAB substrate chromogen solution (DakoCytomation). Slides were counterstained with hematoxylin, dehydrated, and mounted. Each run included, for each patient, phosphate buffered solution (PBS) used as the primary antibody for the negative controls, while samples known to express these markers strongly served as the positive controls. Experienced pathologist reviewed the immunohistochemical slides and immuno-staining intensity results were recorded as 0 for no staining, 1 for faint, 2 for moderate, and 3 for intense staining. Staining percentage of each core from $0 \%$ to $100 \%$ was also recorded. Then, $\mathrm{H}$-score from 0 to 300 was used by multiply staining intensity and percentage of each core. Finally, immunostaining result was classified as negative while $\mathrm{H}$-score lesser than 150 (included) and classified as positive while H-score more than 150.

\section{Statistical analyses}

Statistical analyses were all performed using SPSS-18.0 software (SPSS Inc., Chicago, IL). All data were tested for normal distribution with Kolmogorov-Smirnov test and for homogeneity of variances with Levene's test. To test for differences between positive and negative of immunohistochemical expression, chi-square analysis was performed for categorical variables. To observe the difference between invasion assay results, $t$-test was used. Finally, Kaplan-Meier plot was done and the overall survival was calculated by the log-rank test. In addition, the online tool Kaplan Meier-plotter [24] was also used to generate a validation set of Kaplan-Meier curves based on the expression of p53 and HOXA5. Overall survival of lung cancer patients was stratified using the "Auto select best cutoff" feature. All statistical tests were two-sided and considered statistically significant when $\mathrm{p}<0.05$.

\section{Results}

\section{Clinicopathological features of p53 and HOXA5 expression in non-small cell lung cancer}

The relationship between clinicopathologic features and HOXA5 or p53 expression in NSCLC was summarized in Table 1. Among all characteristics, age and carcinoma cell type showed significant differences in HOXA5 expression. Young age $(<65$ years old) and adenocarcinoma were the two clinical features with higher HOXA5 expression rate $(P=$ 0.009 and $P=0.036$, respectively). However, p53 expression was more frequent in squamous cell carcinoma than adenocarcinoma $(P<0.001)$. No significant associations were observed between HOXA5 or p53 expression and gender or tumor differentiation status. In addition, there was no significant difference in the expression ratio of early stage (stage I and II) and late stage (stage III and IV). This may indicated that HOXA5 involves in the early stage of the lung tumorigenesis. 
Table 1. Chi square test of different clinicopathological parameters and biomarkers of $\mathrm{p} 53$ and HOXA5

\begin{tabular}{|c|c|c|c|c|c|c|}
\hline & P53 & & & HOXA5 & & \\
\hline & $(+)$ & $(-)$ & $\mathrm{P}$ value & $(+)$ & $(-)$ & $\mathrm{P}$ value \\
\hline \multicolumn{7}{|l|}{ Age (year) } \\
\hline$<65$ & $9(29 \%)$ & $22(71 \%)$ & 0.845 & $17(55 \%)$ & $14(45 \%)$ & 0.009 \\
\hline$\geqq 65$ & $12(30 \%)$ & $28(70 \%)$ & & $9(23 \%)$ & $31(77 \%)$ & \\
\hline \multicolumn{7}{|l|}{ Gender } \\
\hline Male & $17(35 \%)$ & $31(65 \%)$ & 0.218 & $15(31 \%)$ & $33(69 \%)$ & 0.239 \\
\hline Female & $4(17 \%)$ & $19(83 \%)$ & & $11(48 \%)$ & $12(52 \%)$ & \\
\hline \multicolumn{7}{|l|}{ Type } \\
\hline SCC & $11(61 \%)$ & $7(39 \%)$ & $<0.001$ & $3(17 \%)$ & $15(83 \%)$ & 0.036 \\
\hline $\mathrm{AdCa}$ & $10(19 \%)$ & $43(81 \%)$ & & $23(43 \%)$ & $30(57 \%)$ & \\
\hline \multicolumn{7}{|c|}{ Differentiation } \\
\hline Well & $1(13 \%)$ & $7(87 \%)$ & 0.406 & $2(25 \%)$ & $6(75 \%)$ & 0.141 \\
\hline Moderate & $12(29 \%)$ & $29(71 \%)$ & & $19(46 \%)$ & $23(54 \%)$ & \\
\hline Poor & $8(36 \%)$ & $14(64 \%)$ & & $5(23 \%)$ & 17 (77\%) & \\
\hline \multicolumn{7}{|l|}{ Stage } \\
\hline I, II & $17(31 \%)$ & $38(69 \%)$ & 0.909 & $21(38 \%)$ & $34(62 \%)$ & 0.927 \\
\hline III, IV & $4(25 \%)$ & $12(75 \%)$ & & $5(31 \%)$ & $11(69 \%)$ & \\
\hline
\end{tabular}

SCC $=$ Squamous cell carcinoma; $\mathrm{AdCa}=$ Adenocarcinoma .

Data are shown as number (percentage).

\section{Expression patterns of HOXA5/p53 and prognosis significance}

HOXA5 is a transcription factor. Therefore, the localization will determine the effect of HOXA5 in the lung cancer tumorigenesis. As shown in Figure 1, the HOXA5 protein expression was detected mostly in the nuclei of the non-tumor part using immunohistochemical stain $(P<0.05)$. On the contrary, the expression pattern of HOXA5 protein in the tumor part was mainly cytoplasmic staining pattern $(54.1 \%)$ or even lost its expression $(31.3 \%)$. The expression details were shown in the Table S1. Unlike HOXA5, the expression of p53 was only in the nuclei in all detected NSCLC specimens (Figure 1A). We then investigated the prognostic significances of p53 and HOXA5 expression status in NSCLCs by log-rank test of Kaplan-Meier plot. As we know the expression of p53 protects cells against malignant transformation, we further subgrouped all the study subjects into three groups [p53 (+) with regardless of HOXA5 expression, p53 (-) and HOXA5 (+), and p53 (-) and HOXA5 (-)] according to their different staining patterns. Representative images of HOXA5/ p53-positive and -negative NSCLC specimens were shown in Figure 2A. As shown in Figure 2B, the patients within the p53 (-) and HOXA5 (-) group had a significantly poorer overall survival than the other two groups $(P=0.033)$. We further validated the prognostic value of p53 and HOXA5 mRNA expression in lung cancer patients by using the Kaplan Meier-plotter online database (GSE50081 cohort; $\mathrm{n}=181$ ). Group with high mRNA expression levels of both p53 and HOXA5 is associated with better overall survival in lung cancer patients, $\mathrm{HR}=0.46 \quad(0.29-0.75), \quad P=0.0015 \quad$ (Figure 2C). Our patient's specimen results were consistent with the online database.

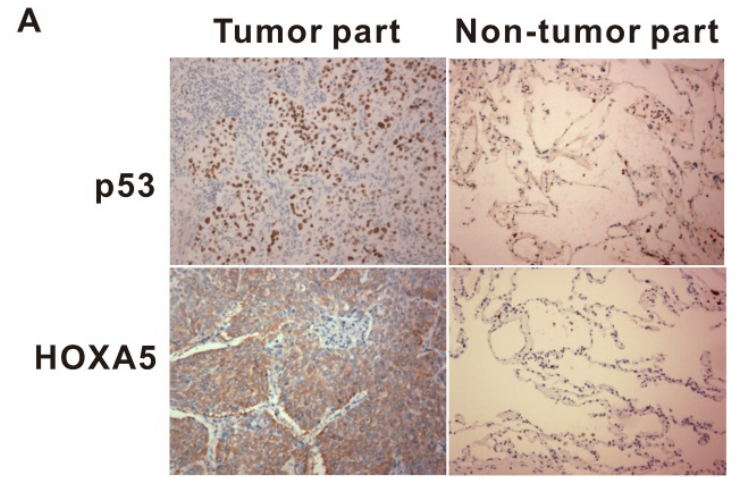

B

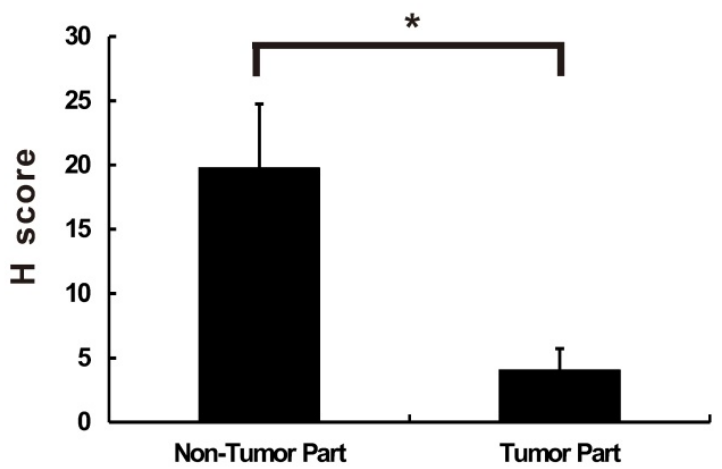

C

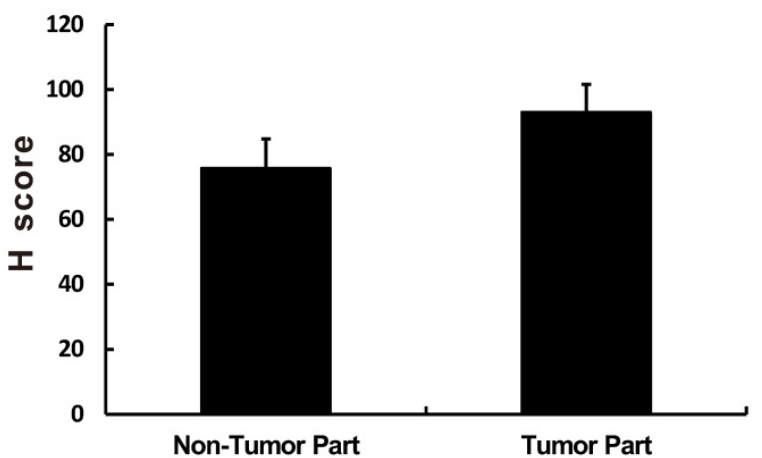

Figure 1. The immunoreactive location of p53 and HOXA5 in non-small cell lung cancer. The immunoreactive location of $\mathrm{p} 53$ and $\mathrm{HOXA} 5$ are different in non-small cell lung cancer. (A) The expression location of $\mathrm{p} 53$ is mainly in the nuclei while HOXA5 expressed mainly in the cytoplasm of the tumor part. All pictures were taken in $400 \mathrm{X}$. The HOXA5 $\mathrm{H}$-score of nuclear staining is significantly lower in tumor part (B) while no significant difference for cytoplasmic staining pattern in both tumor and non-tumor part (C). $*, P<0.05$.

\section{Effects of HOXA5 and various p53 constructs on $\mathrm{H} 1299$ cell invasion}

In our previous study, we found that HOXA5 expression could significantly inhibit human lung adenocarcinoma CL1-5 cell migration and invasion capabilities [18]. However, whether the cooperation between HOXA5 and p53 could enhance this inhibition effect is still unknown. To determine the 
effects of combined HOXA5 and p53 overexpression on lung cancer cell invasion, H1299 cells, a p53-null cell line was chosen. First, various human p53 cDNA wild-typed or mutated expression vectors or control vectors were transiently transfected into H1299 cells and performed in vitro invasion assays. As shown in Figure 3A, wild-typed p53 expression could significantly inhibit H1299 cell invasive capabilities compared with pCEP4 vector control while p53 R248W mutant increases H1299 cell invasion. Then, HOXA5 expression or control vector was either transiently transfected or cotransfected with various p53 expression constructs into H1299 cells and conducted invasion assays. Consistence with previous results, there was a significant decrease in the invasive potential of the HOXA5-transfected H1299 cells when compared with vector control transfectants. In addition, cotransfection of HOXA5 and wild-typed p53, p53 V173L or p53 L22Q/W23S mutated expression constructs could further lower the invasive capabilities of H1299 cells (Figure 3B). To further address the effect of HOXA5 expression on p53-mutated lung cancer cell invasive capability, NCI-H322M (p53 R248L) and NCI-H522 (p53 P191 frameshift) cells were transiently transfected with HOXA5 expression or control vectors to perform the invasion assays (Figure S1). Overexpression of HOXA5 could significantly inhibit both lung cancer cells invasive capabilities regardless of p53 genetic background $(P<0.05)$.

\section{The cooperative effects of HOXA5 and p53 on MMP2 activity}

Matrix metalloproteinases (MMPs) have been found to play an important role in lymphatic and vascular invasion of lung cancer [25]. In addition, p53 is known to modulate MMP2 expression [26]. We cotransfected HOXA5 and p53 expression vectors in different concentrations and determined the invasive potential of H1299 transfectants. The results showed that overexpression of both HOXA5 and p53 could inhibit the H1299 cell invasive capabilities in a concentration-dependent manner (Figure 4A). Moreover, to determine whether MMPs are activated by HOXA5 and p53, we used gelatin zymography to assess the enzyme activity of MMPs in HOXA5/p53 cotransfected cells. The results revealed that overexpression of HOXA5 and p53 significantly decreased the activity of MMP2 also in a concentration-dependent manner, while that of MMP9 was unaltered (Figure 4B).

\section{Discussion}

The results of this study showed that the expression of HOXA5 in NSCLC was associated with different lung cancer subtype, higher expression ratio in adenocarcinoma than squamous cell carcinoma in human specimens. Moreover, HOXA5 expressed mainly in the cytoplasm or loss its expression rather than that in the nuclei of the NSCLC tumor part. This indicates that HOXA5 may loss its function as being a transcription factor of a tumor suppressor gene. Furthermore, our data showed that overexpression of HOXA5 could reduce invasion abilities in different lung cancer cell lines via a p53-independent pathway. HOXA5-overexpressing H1299 cells transfected with wild type p53 further decreased invasive ability and had a concentration-dependent effect. The suppressed invasive ability of HOXA5-overexpressing cells would be partly due to the inhibition of MMP-2 activity. When applied p53 and HOXA5 as prognostic factor for NSCLC patients, poorest prognosis is seen in group with both non-immunoreactive for p53 and HOXA5. To further confirm our findings, the Kaplan Meier-plotter online database (GSE50081 cohort; $\mathrm{n}=181$ ) was applied. Group with high mRNA expression levels of both p53 and HOXA5 is associated with better overall survival in lung cancer patients which was consistent with our results. In summary, HOXA5 inhibits tumor cell invasion partly by decreasing MMP-2 activity and could serve as a good prognostic factor in NSCLC especially when p53 loss of function.

Previous study identified consensus HOX binding sites in the p53 promoter and HOXA5 could activate p53 transcriptional expression [22]. Expression of HOXA5 in cancer cells expressing wild-type p53, but not in isogenic variants lacking the p53 gene, led to apoptotic cell death [22]. Moreover, loss of HOXA5 expression could result in Twist activation and cause aberrant cell cycle regulation which finally promoted the breast tumorigenesis [27]. Using a p53-promoter-reporter system, HOXA5 could partially restore the inhibitory effects of Twist on p53 target genes. In summary, these data showed that loss of HOXA5 expression in human breast cancer may lead to the loss of p53 expression. However, it seems to have another mechanism in NSCLC according to our results. Squamous cell carcinoma showed higher p53 expression ratio but the HOXA5 expression is higher in adenocarcinoma. Furthermore, regardless of HOXA5 expression, expression of p53 in NSCLC have better prognosis. In the animal model, the presence of one Hoxa5 mutant allele had no impact on mammary tumor formation. In contrast, the complete loss of Hoxa5 function influenced the tumorigenic outcome of p53 (+/-) mammary glands. However, the collaborative nature of this interaction did not depend on the transcriptional regulation of p53 by Hoxa5 [28]. 
A

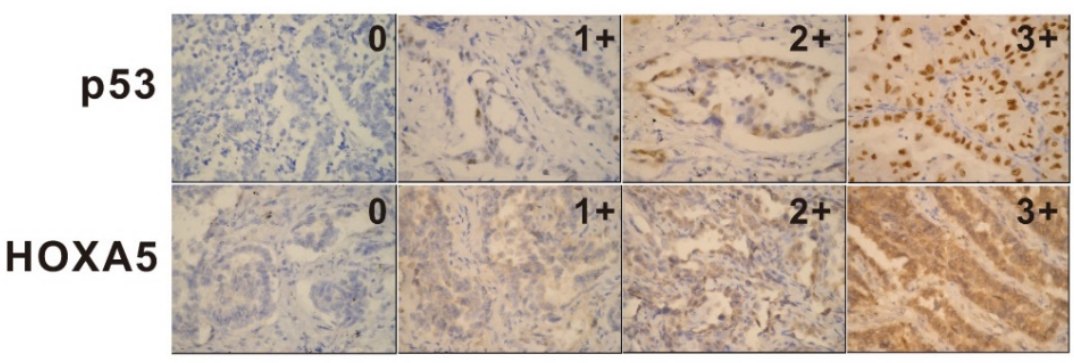

B

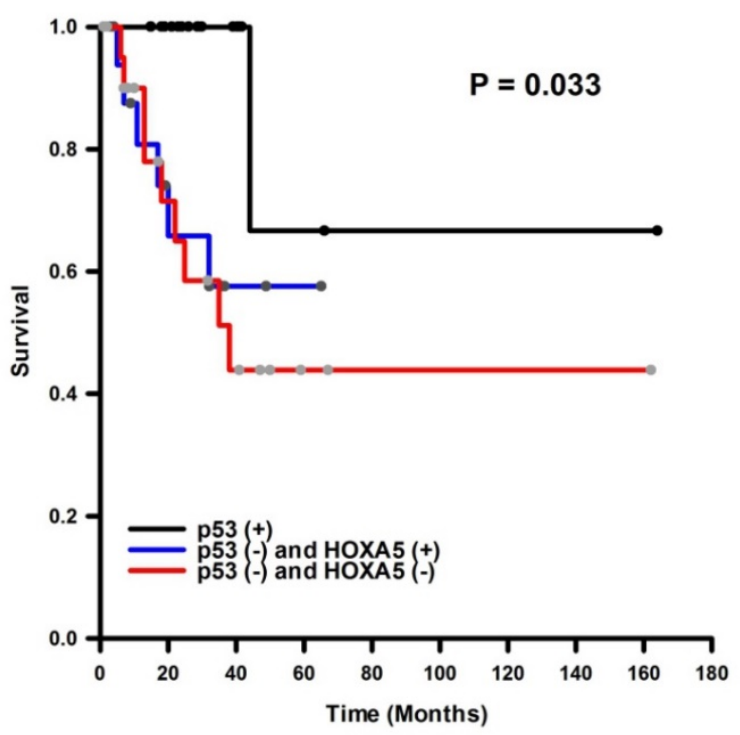

C

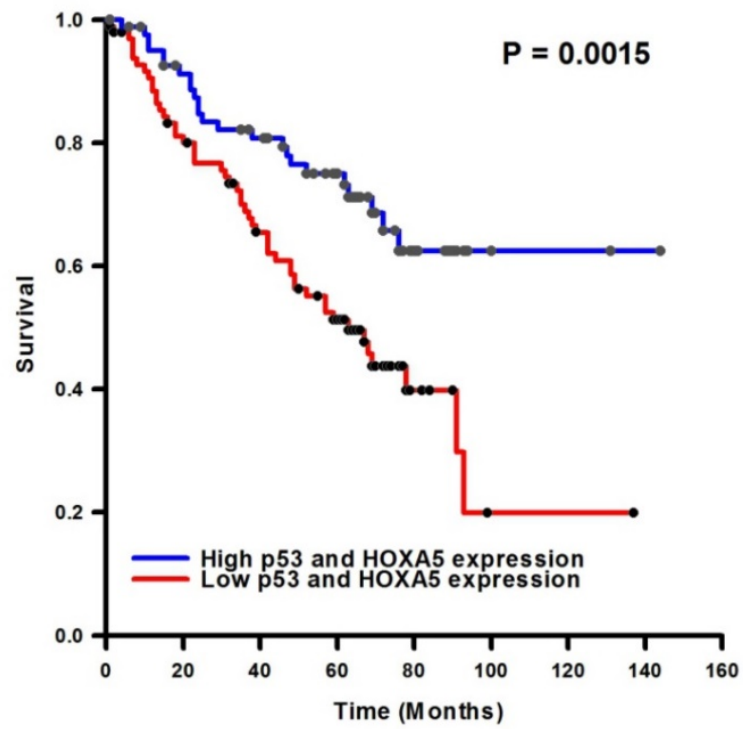

Figure 2. The p53 and HOXA5 expression levels and clinical outcome correlations. (A) The expression intensity examples of p53 and $\mathrm{HOXA5}$ in non-small cell lung cancer. The intensity is from non-expression (0) to highest expression intensity $(3+)$. The expression location of p 53 is mainly in the nuclei while HOXA5 expressed mainly in the cytoplasm. All pictures were taken in 400X. (B) Kaplan-Meier plot of overall survival by different expression status of $p 53$ and HOXA5. $\mathrm{p} 53(+)=$ group with cases were immunoreactive for $\mathrm{p} 53$ and regardless of HOXA5 immunoexpression. p53 (-) and HOXA5 (+) = group with cases were non-immunoreactive for p53 and cytoplasmic immunoreactive for HOXA5. p53 (-) and HOXA5 (-) = group with all cases were both non-immunoreactive for $\mathrm{p} 53$ and HOXA5. (C) The prognostic value of $\mathrm{p} 53$ and HOXA5 expressions in lung cancer patients (GSE50081 cohort; $\mathrm{n}=181$ ). The desired Affymetrix ID is valid: 201746 at (p53) and 213844_at (HOXA5). Data was analyzed by using Kaplan-Meier plotter. 
A

H1299 Vector WT R248W V173L L22Q/W23S

\begin{tabular}{|l|l|l|l|l|}
\hline & & & & \\
& & & &
\end{tabular}

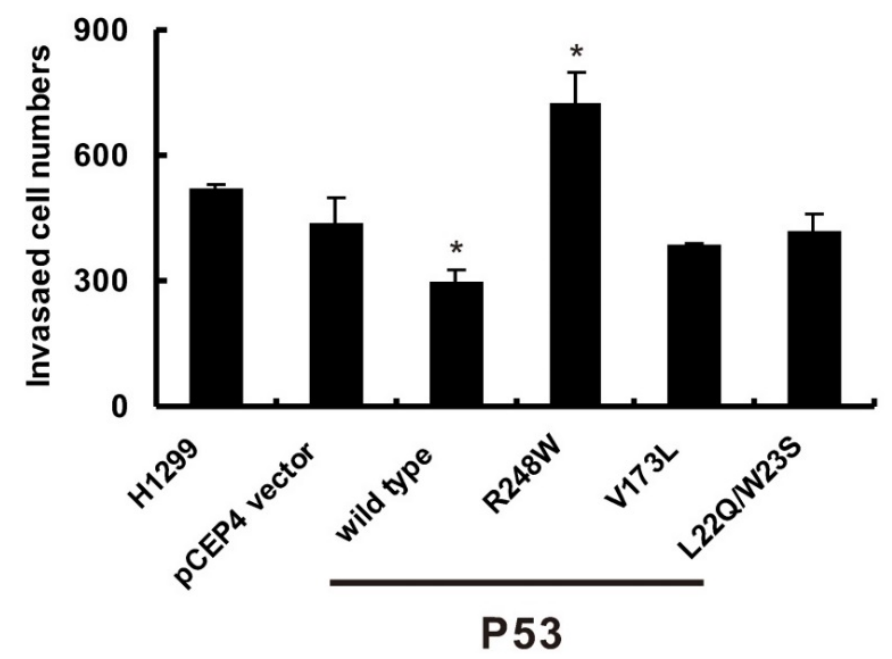

B

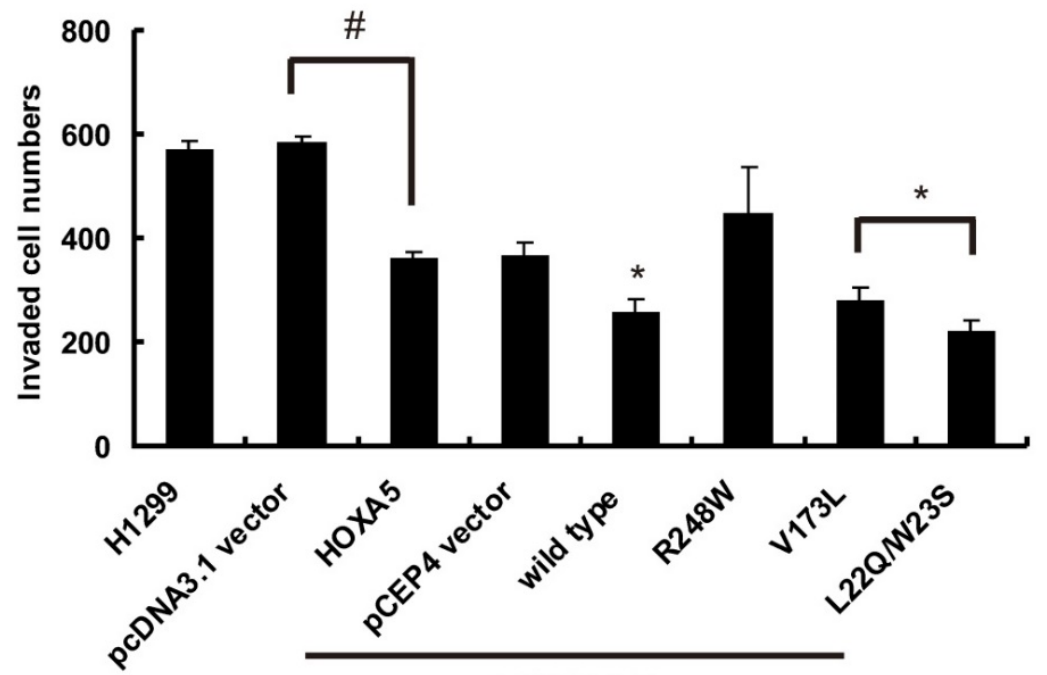

HOXA5

P53

Figure 3. HOXA5 overexpression and different $\mathbf{p} 53$ mutants co-transfection could change cell invasive abilities in $\mathrm{H} 1299$ cells. The influence of different $\mathrm{p} 53$ mutants on invasion activity in H1299 cells. (A) Upper panel, representative images of invasion assay. Lower panel, densitometry evaluation for invasive cells. Means of treatments are significantly different from vector control group (each experiment was performed in triplicate), mean \pm SD; $*, P<0.05$. (B) When we transfected HOXA5 in H1299 cells, the invaded cells were decreased about one-thirds as compared with pcDNA3.1 vector $(\#, P<0.05)$. Then we transfected different $\mathrm{p} 53$ mutants into stably HOXA5- expressing H1299 cells to determine the cell invasive abilities. Each experiment was performed in triplicate, mean $\pm \mathrm{SD} ; *, P<0.05$, compared to $\mathrm{PCEP} 4$ vector control. 

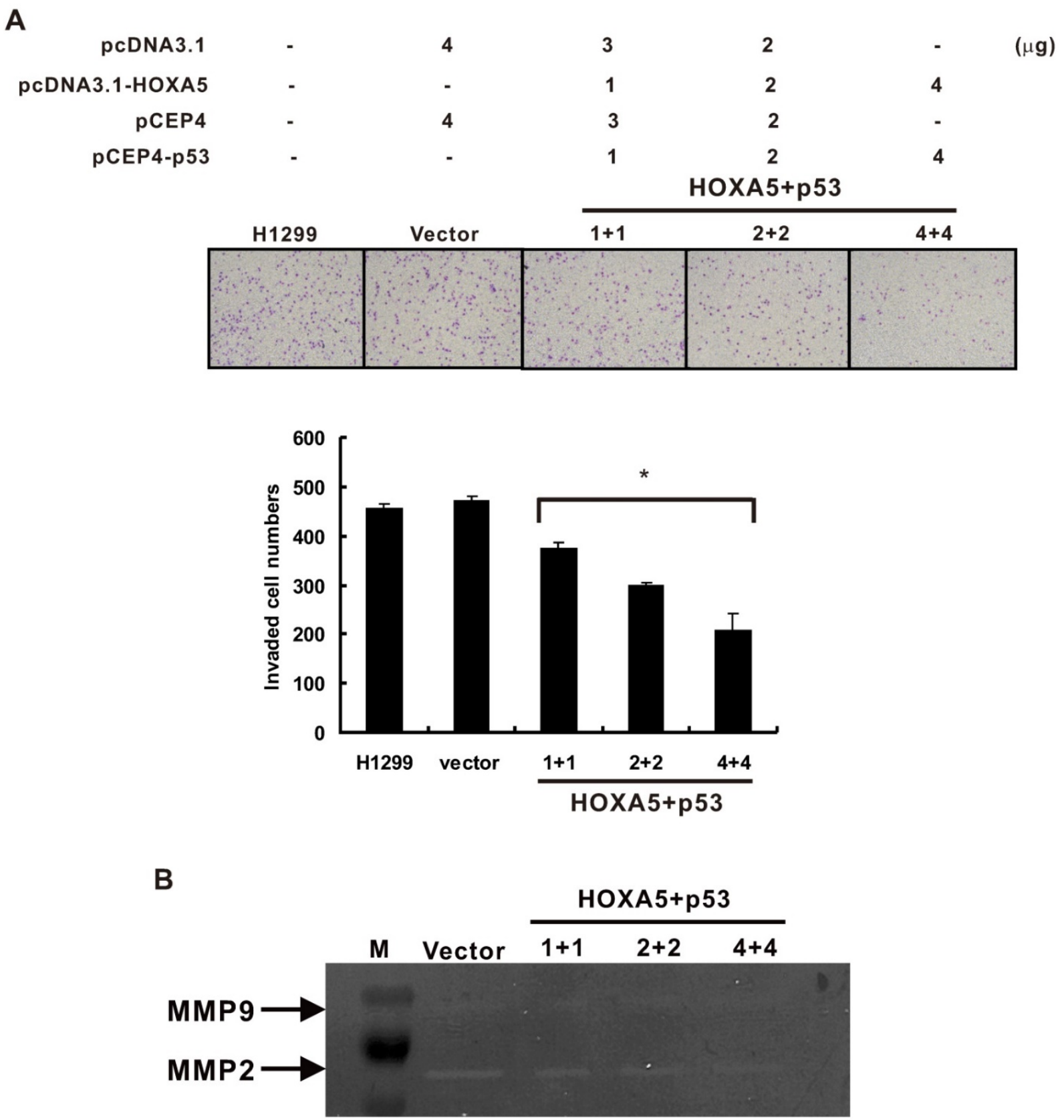

Figure 4. Co-expression of HOXA5 and wild type p53 could cooperatively inhibit invasion ability and decrease MMP-2 activity in H1299 cells. (A) Upper panel, representative images of invasion assay. Lower panel, densitometry evaluation for invasive cells. Means of treatments are significantly different from vector control group. Each experiment was performed in triplicate, mean \pm SD; *, $P<0.05$. (B) Zymography assay. Means of treatments are significantly different from vector group. Each experiment was performed in duplicate.

Our results showed different invasion abilities in different p53 mutant genotypes of H1299 cells. For example, to study the possible role for the p53 N-terminus, a doubly mutated form of human p53, L22Q/W23S, was examined. Previous studies showed that these transactivation domain residues may interact with a number of proteins including Mdm2, adenovirus E1B [29], TAFs [30] and p300 [31]. In addition, p53-V173L, p53-R175H and p53-H179Q are commonly found in human cancers [32]. Moreover, several p53 hotspot mutations (R175H, R248W and $\mathrm{R} 273 \mathrm{H}$ ) were found not only lost p53-dependent tumor suppressor activities, but also acquired new oncogenic activities to promote progression of cancers [33]. When HOXA5 was transfected into the H1299 cell, the invasion ability was decreased in about one third of the total number of invasive cells. Even under the induction of p53 mutant proteins in H1299 cells, HOXA5 overexpression could significantly lower cancer cell invasive capabilities. These results suggested that after overexpression of HOXA5 might activate or suppress the expression levels of some downstream target genes and these effects could overcome the influences of various p53 mutants in the 
lung cancer cells. Moreover, other p53 mutant cell lines (H522 and H322M) transfected with HOXA5 still keep its ability to decrease invasion capabilities. These results are consistent with our previous study using different cell lines of A549, PE089 and CL1-5 cells in invasion assays [18].

To further explore the invasion-related targets regulated by HOXA5 and p53, MMP-2 and MMP-9 were detected. MMP-2 expression level was decreased in a concentration-dependent manner when transfected with HOXA5 and wild type p53 expression vectors. Although previous study showed that p53 could modulate the expression of MMP2 [26], whether HOXA5 could regulate MMP2 expression is unclear so far. We predicted the potential transcription factor binding sites in MMP2 promoter and found several HOXA5 binding sites were located in it. Our preliminary data suggested that HOXA5 could decrease MMP-2 mRNA expression levels in a concentration-dependent manner (data not shown). Overexpression of HOXA5 and p53 significantly decreased the activity of MMP2 in a concentration-dependent manner, while that of MMP9 was unaltered. Although further study needs to clarify the roles of HOXA5 and MMP2 promoter regulation, it provides some clues that HOXA5 could cooperate with p53 in cancer cell invasion inhibition through modulating MMP2 expression and activity. To our knowledge, this is the first study demonstrate the association between HOXA5 and tumor invasion with and without p53 mutation in NSCLC.

HOXA5 is normally expressed in the nuclei as a transcription factor. In the studies done by Boucherat et al. and Garin et al., the immunostaining pattern of HOXA5 was located in the pulmonary epithelium nuclei during the pulmonary development [12,34]. However, another study found different expression pattern in the pulmonary malignancy. By immunohistochemical analysis, HOXA5 was localized in the cytoplasm of tumor cells in both adenocarcinoma and squamous cell carcinoma tissues with different expression levels [16]. These results suggest that the disordered patterns of HOX gene expressions were involved not only in the development of NSCLC but also in the histologically aberrant diversity of NSCLC types [16]. Similar result was also found in the breast cancer. Loss expression of HOXA5 nuclear staining in invasive carcinoma was seen when compared with normal breast tissue. Our data showed similar results that HOXA5 located mostly in the nuclei of the non-tumor part but mainly in cytoplasm of the tumor part. HOX proteins have been known to have other functions than serving as a transcription factor [16]. For example, the homeodomain of HOX proteins might bind to other proteins and modulate their activities [35]. Therefore, we could not rule out the possibility that HOXA5 might interact with other proteins in the cytoplasm, interfere with the translocation of HOXA5 into nucleus and loss its transcriptional role in the lung tumor tissue. However, this issue should be addressed in the further studies.

Nearly $70 \%$ of breast cancer decreased HOXA5 protein levels when compared to normal breast tissues. However, the survival rate amongst the different low levels of HOXA5 expressing breast tumors was not significant [36]. Not only in breast cancer, Kim et al. found that there was no influence of HOXA5 methylation on survival in all NSCLC or at stages II-IV. However, in the patients with stage I NSCLC, HOXA5 methylation was associated with a borderline significance of poor survival [17]. On the contrary, oral squamous cell carcinoma patients presenting lower expression of HOXA5 had poorer prognosis when compared to those with higher expression [37]. Our results supported that HOXA5 would be a prognostic factor if p53 was considered in the study population. Lower expression of HOXA5 could serve as a poor prognostic factor mainly in p53 non-immunoreactive NSCLC patients. Recent studies reported that three miroRNAs, miR-196a, miR-130, and miR-1271, could regulate NSCLC cell proliferation, invasion, migration, and angiogenesis via the downregulation of HOXA5 [38-40]. Thus, these microRNAs might represent potential therapeutic targets for NSCLC intervention.

The strength of the current study is the first study evaluates the correlation between HOXA5 and MMP in NSCLC. In addition, the role of p53 status involved in HOXA5-mediated cancer cell invasive inhibition is also considered in the current study. Finally, follow-up was also done for further validate the clinical significance of the HOXA5 in NSCLC with or without p53 expression. Still, there are several limitations in the present study. First, no genetic mutation tests were done. Therefore, it cannot be seen the correlation between HOXA5 and other mutation such as EGFR mutation and ALK translocation. However, in our previous study we found that HOXA5 expression was associated with better clinical outcome in NSCLC patients with wild-type EGFR [18]. In addition, methylation of the HOXA5 gene was seen in some cancers and we did not perform the methylation tests in the current study. According to the report done by Kim et al., HOXA5 methylation might be observed in about $81.3 \%$ of the NSCLC and correlated with gene expression which is supported by RT-PCR and immunohistochemical analysis [17]. Therefore, this influence may be low for us to realize the inhibition ability of HOXA5 in tumor invasion and 
correlate the HOXA5 expression levels in prognosis. At last, although there are follow-up data in the present study, relative small cases number and short follow-up periods may limit the interpretation of the results. However, we adapted another online data of 181 NSCLC patients to validate our findings which may also minimize the bias.

In conclusion, our results showed that overexpression of HOXA5 could reduce invasion abilities in several lung cancer cell lines via a p53-independent pathway. Furthermore, HOXA5-overexpressing H1299 cells transfected with wild type p53 could further decrease invasive ability and has a concentration-dependent effect. The suppressed invasive ability of HOXA5overexpressing cells would be partly due to the inhibition of MMP-2 activity. Therefore, loss expression of both p53 and HOXA5 in human NSCLC specimen showed poorest prognosis when compared with either p53 or HOXA5 expression groups. All these efforts will provide the clues to interpret the molecular mechanisms of HOXA5 and p53 are invasion suppressors and could develop targeted-therapeutic strategies in NSCLC.

\section{Supplementary Material}

Supplementary figure and table. http://www.jcancer.org/v08p1071s1.pdf

\section{Acknowledgements}

This study was supported by grants from the Shin Kong Wu Ho-Su Memorial Hospital, Taiwan, R.O.C. (99-SKH-FJU-13 to CJC and CCW), as well as by grants from the National Science Council (NSC) and Ministry of Science and Technology, Taiwan, R.O.C. (NSC102-2314-B-030-005 and MOST 104-2320-B-030-010 to CCW). The funders had no role in study design, data collection and analysis, decision to publish, or preparation of the manuscript.

\section{Competing Interests}

The authors have declared that no competing interest exists.

\section{References}

1. Vansteenkiste J, De Ruysscher D, Eberhardt WE, et al. Early and locally advanced non-small-cell lung cancer (NSCLC): ESMO Clinical Practice Guidelines for diagnosis, treatment and follow-up. Ann Oncol. 2013; 24 (Suppl 6):vi89-98.

2. Siegel R, Ma J, Zou Z, Jemal A. Global cancer statistics, 2014. CA Cancer J Clin. 2014; 64: 9-29.

3. Detterbeck FC, Mazzone PJ, Naidich DP, Bach PB. Screening for lung cancer: Diagnosis and management of lung cancer, 3rd ed: American College of Chest Physicians evidence-based clinical practice guidelines. Chest. 2013; 143 (5 Suppl):e78S-92S

4. Alberg AJ, Brock MV, Ford JG, Samet JM, Spivack SD. Epidemiology of lung cancer: Diagnosis and management of lung cancer, 3rd ed: American College of Chest Physicians evidence-based clinical practice guidelines. Chest. 2013; 143 (5 Suppl):e1S-29S.
5. Peters S, Adjei AA, Gridelli C, Reck M, Kerr K, Felip E. Metastatic non-small-cell lung cancer (NSCLC): ESMO Clinical Practice Guidelines for diagnosis, treatment and follow-up. Ann Oncol. 2012; 23 (Suppl 7):vii56-64.

6. Gould MK, Donington J, Lynch WR, et al. Evaluation of individuals with pulmonary nodules: when is it lung cancer? Diagnosis and management of lung cancer, 3rd ed: American College of Chest Physicians evidence-based clinical practice guidelines. Chest. 2013; 143 (5 Suppl):e93S-120S.

7. Schwartz AM, Rezaei MK. Diagnostic surgical pathology in lung cancer: Diagnosis and management of lung cancer, 3rd ed: American College of Chest Physicians evidence-based clinical practice guidelines. Chest. 2013; 143 (5 Suppl):e251S-62S.

8. Volpe MV, Wang KT, Nielsen HC, Chinoy MR. Unique spatial and cellular expression patterns of Hoxa5, Hoxb4, and Hoxb6 proteins in normal developing murine lung are modified in pulmonary hypoplasia. Birth Defects Res A Clin Mol Teratol. 2008; 82: 571-84.

9. Mandeville I, Aubin J, LeBlanc M, et al. Impact of the loss of Hoxa5 function on lung alveogenesis. Am J Pathol. 2006; 169: 1312-27.

10. Kinkead R, LeBlanc M, Gulemetova R, et al. Respiratory adaptations to lung morphological defects in adult mice lacking Hoxa5 gene function. Pediatr Res. 2004; 56: 553-62.

11. Boucherat $\mathrm{O}$, Montaron $\mathrm{S}$, Berube-Simard FA, et al. Partial functional redundancy between Hoxa5 and Hoxb5 paralog genes during lung morphogenesis. Am J Physiol Lung Cell Mol Physiol. 2013; 304: L817-30.

12. Boucherat $\mathrm{O}$, Chakir J, Jeannotte $\mathrm{L}$. The loss of Hoxa5 function promotes Notch-dependent goblet cell metaplasia in lung airways. Biol Open. 2012; 1: 677-91.

13. Yoo KH, Park YK, Kim HS, Jung WW, Chang SG. Epigenetic inactivation of HOXA5 and MSH2 gene in clear cell renal cell carcinoma. PatholInt. 2010; 60: 661-6.

14. Watson RE, Curtin GM, Hellmann GM, Doolittle DJ, Goodman JI. Increased DNA methylation in the HoxA5 promoter region correlates with decreased expression of the gene during tumor promotion. Mol Carcinog. 2004; 41: 54-66.

15. Loh M, Liem N, Vaithilingam A, et al. DNA methylation subgroups and the $\mathrm{CpG}$ island methylator phenotype in gastric cancer: a comprehensive profiling approach. BMC gastroenterol. 2014; 14: 55.

16. Abe M, Hamada J, Takahashi O, et al. Disordered expression of HOX genes in human non-small cell lung cancer. Oncol Rep. 2006; 15: 797-802.

17. Kim DS, Kim MJ, Lee JY, et al. Epigenetic inactivation of Homeobox A5 gene in nonsmall cell lung cancer and its relationship with clinicopathological features. Mol Carcinog. 2009; 48: 1109-15.

18. Wang CC, Su KY, Chen HY, et al. HOXA5 inhibits metastasis via regulating cytoskeletal remodelling and associates with prolonged survival in non-small-cell lung carcinoma. PLoS One. 2015; 10: e0124191.

19. Boudreau HE, Casterline BW, Burke DJ, Leto TL. Wild-type and mutant p53 differentially regulate NADPH oxidase 4 in TGF-beta-mediated migration of human lung and breast epithelial cells. Br J Cancer. 2014; 110: 2569-82.

20. Brambilla E, Gazzeri S, Lantuejoul S, et al. p53 mutant immunophenotype and deregulation of p53 transcription pathway (Bcl2, Bax, and Waf1) in precursor bronchial lesions of lung cancer. Clin Cancer Res. 1998; 4: 1609-18.

21. Campling BG, el-Deiry WS. Clinical implications of p53 mutations in lung cancer. Methods Mole Med. 2003; 75: 53-77.

22. Raman V, Martensen SA, Reisman D, et al. Compromised HOXA5 function can limit p53 expression in human breast tumours. Nature. 2000; 405: 974-8.

23. Kim EM, Park JK, Hwang SG, et al. Nuclear and cytoplasmic p53 suppress cell invasion by inhibiting respiratory Complex-I activity via Bcl-2 family proteins. Oncotarget. 2014; 5: 8452-65.

24. Gyorffy B, Surowiak P, Budczies J, Lanczky A. Online survival analysis software to assess the prognostic value of biomarkers using transcriptomic data in non-small-cell lung cancer. PLoS One. 2013; 8: e82241.

25. Guo CB, Wang S, Deng C, Zhang DL, Wang FL, Jin XQ. Relationship between matrix metalloproteinase 2 and lung cancer progression. Mol Diagn Ther. 2007; 11: 183-92.

26. Bian J, Sun Y. Transcriptional activation by p53 of the human type IV collagenase (gelatinase A or matrix metalloproteinase 2) promoter. Mol Cell Biol. 1997: 17: 6330-8.

27. Stasinopoulos IA, Mironchik Y, Raman A, Wildes F, Winnard P, Jr., Raman V. HOXA5-twist interaction alters p53 homeostasis in breast cancer cells. J Biol Chem. 2005; 280: 2294-9.

28. Gendronneau G, Lemieux M, Morneau M, et al. Influence of Hoxa5 on p53 tumorigenic outcome in mice. Am J Pathol. 2010; 176: 995-1005.

29. Lin J, Chen J, Elenbaas B, Levine AJ. Several hydrophobic amino acids in the p53 amino-terminal domain are required for transcriptional activation, binding to mdm-2 and the adenovirus 5 E1B $55-\mathrm{kD}$ protein. Genes \& Dev. 1994; 8: 1235-46.

30. Lu H, Levine AJ. Human TAFII31 protein is a transcriptional coactivator of the p53 protein. Proc. Natl. Acad. Sci. USA 1995; 92: 5154-8.

31. Gu W, Roeder RG. Activation of p53 sequence-specific DNA binding by acetylation of the p53 C-terminal domain. Cell. 1997; 90: 595-606.

32. Salah Z, Haupt S, Maoz M, et al. p53 controls hPar1 function and expression. Oncogene. 2008; 27: 6866-74.

33. Liu DP, Song $\mathrm{H}, \mathrm{Xu} \mathrm{Y}$. A common gain of function of p53 cancer mutants in inducing genetic instability. Oncogene. 2010; 29: 949-56.

34. Garin E, Lemieux M, Coulombe Y, Robinson GW, Jeannotte L. Stromal Hoxa5 function controls the growth and differentiation of mammary alveolar epithelium. Dev Dyn. 2006; 235: 1858-71. 
35. Shen WF, Krishnan K, Lawrence HJ, Largman C. The HOX homeodomain proteins block CBP histone acetyltransferase activity. Mol Cell Biol. 2001; 21: 7509-22.

36. Henderson GS, van Diest PJ, Burger H, Russo J, Raman V. Expression pattern of a homeotic gene, HOXA5, in normal breast and in breast tumors. Cell Oncol. 2006; 28: 305-13.

37. Rodini $\mathrm{CO}$, Xavier FC, Paiva KB, et al. Homeobox gene expression profile indicates HOXA5 as a candidate prognostic marker in oral squamous cell carcinoma. Int J Oncol. 2012; 40: 1180-8.

38. Liu XH, Lu KH, Wang KM, et al. MicroRNA-196a promotes non-small cell lung cancer proliferation and invasion through targeting HOXA5. BMC Cancer. 2012; 12: 348.

39. Chen Y, Gorski DH. Regulation of angiogenesis through a microRNA (miR-130a) that down-regulates antiangiogenic homeobox genes GAX and HOXA5. Blood. 2008; 111: 1217-26.

40. Wang $\mathrm{Y}, \mathrm{Xu} \mathrm{L}$, Jiang L. miR-1271 promotes non-small-cell lung cancer cell proliferation and invasion via targeting HOXA5. Biochem Biophys Res Commun. 2015; 458: 714-9. 